\title{
Formación de maestros para la educación infantil en la Universidad Pedagógica Nacional de Colombia: una aproximación desde los temas y objetos de la producción investigativa
}

Teacher Training for Early Childhood Education at Universidad Pedagógica Nacional de Colombia: An Approach from Topics and Objects of Research Production

\section{Carolina Soler Martín*}

(iD https://orcid.org/0000-0003-4380-4589

$$
\text { Yeimy Cárdenas Palermo** }
$$

(iD https://orcid.org/0000-0003-1869-4074

\section{Sandra Marcela Durán Chiappe *** \\ (D) https://orcid.org/0000-0002-1088-3164}

\section{Tipo de Artículo: Informes de Investigación y ensayos inéditos}

Doi: 10.17533/udea.unipluri.20.2.017

Soler Martín, C., Cárdenas Palermo, Y., y Durán Chiappe, S. M. (2020). Formación de maestros para la educación infantil en la Universidad Pedagógica Nacional de Colombia: una aproximación desde los temas y objetos de la producción investigativa. Uni-Pluriversidad, 20(2), e20202017. doi: 10.17533/udea.unipluri.20.2.017

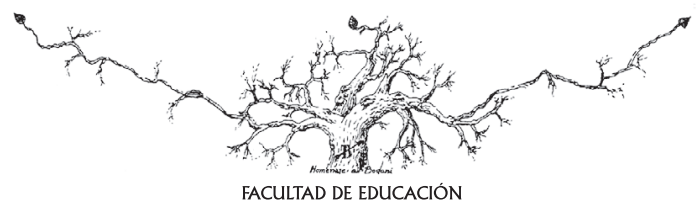

Recibido: 2020-02-15 • Aprobado: 2020-12-22

* Universidad Pedagógica Nacional. Colombia

Email: csmartin@pedagogica.edu.co

** Universidad Pedagógica Nacional. Colombia Email: ypalermo@pedagogica.edu.co

*** Universidad Pedagógica Nacional. Colombia Email: smduran@pedagogica.edu.co 


\title{
Resumen
}

Este artículo es el resultado de una investigación que presenta aproximaciones al estado actual de la discusión sobre la formación de maestros para la educación infantil, desde la producción investigativa de la Universidad Pedagógica Nacional de Colombia. En este estudio de tipo documental y con alcance descriptivo se revisaron como fuentes trabajos de pregrado, tesis de maestría e informes de investigación a partir de dos criterios: el primero, el período de publicación (2014-2019) y el segundo, los temas y objetos de estudio. El análisis de contenido de estas fuentes permitió identificar desarrollos analíticos en tres tendencias: aproximaciones al pensamiento, saberes y concepciones de los educadores infantiles en formación y en ejercicio; prácticas pedagógicas y la formación de profesionales reflexivos; y miradas críticas al currículo de la licenciatura en educación infantil y a otros programas de formación. Los hallazgos derivados de esta investigación esbozan posibles horizontes para la reflexión y el debate alrededor de la formación de educadores infantiles en Colombia.

Palabras clave: formación de maestros, educación inicial, educación infantil.

\begin{abstract}
This article is the result of a research that presents approaches to the current state of the discussion on teacher training for early childhood education, according to the research production of Universidad Pedagógica Nacional de Colombia. In this documentary study with a descriptive scope, undergraduate research, master's thesis and research reports were the sources reviewed under two criteria: time of publication (2014-2019), and topics and study objects. Content analysis allowed to identify analytical developments in three trends: approaches to the thinking, knowledge and conceptions by early childhood educators, both in training and in practice; pedagogical practices and training of reflective professionals; and critical look at the curriculum of the degree in early childhood education and other training programs. The findings outline possible horizons to think and debate around the training of early childhood educators in Colombia.
\end{abstract}

Keywords: teacher training, initial education, early childhood education. 


\section{INTRODUCCIÓN}

口

La pregunta por la formación de maestros para la educación inicial articuló, durante los años 2018 y 2019, una iniciativa de investigación de la Red Educativa Universitaria de Conocimiento y Acción Regional-Reducar, con participación de las universidades pedagógicas de Colombia, Argentina, Honduras, Ecuador, México y Cuba. La Universidad Pedagógica Nacional de Colombia (UPN-Colombia) participó en el diseño y desarrollo del proyecto interinstitucional con financiación del Centro de investigaciones-CIUP (código Reducar-2018). De ese gran interrogante, uno de los objetivos específicos se centró en el análisis de las discusiones recientes acerca de la formación en cada institución.

El artículo que aquí se presenta deriva del objetivo específico del estudio enunciado en el interrogante anterior. El grupo de investigación propuso aproximarse a la formación de maestros para la educación inicial a partir de la producción investigativa de la UPN-Colombia en el periodo 2014-2019 y aportar a la discusión que ha tomado fuerza en Colombia y otros países de la región en torno a la formación inicial, permanente y avanzada de los educadores infantiles y, en esta, los lugares que ocupa la enseñanza, la pedagogía y la didáctica, el reconocimiento social, la comprensión del educador infantil como sujeto reflexivo, político y productor de cultura y, los aportes y discusiones en las políticas públicas para la infancia, particularmente en las últimas dos décadas. Este debate tiene cercanía con declaraciones internacionales sobre la formación de maestros que indican la necesidad de mejorar y ampliar el desarrollo profesional de quienes llevan adelante la educación inicial (OEI, 2000; Orealc/Unesco, 2016).

\section{Metodología}

El análisis presentado es el resultado de una investigación de tipo documental cualitativa con alcance descriptivo. Se consideraron como fuentes documentales trabajos de grado y tesis de maestría publicadas en el repositorio virtual institucional, e informes de investigación resultado de proyectos financiados por el Centro de investigaciones-CIUP. La masa documental se delimitó por criterios de período de publicación (2014-2019) y de delimitación temática, de acuerdo con los objetivos de la investigación -formación de maestros para la infancia-, aunque no constituyera el propósito central de cada fuente documental. Se seleccionaron doce trabajos de grado (pregrado), tres tesis de maestría y cinco informes de proyectos de investigación. Los 20 documentos fueron interrogados en cuanto a sus objetos de estudio, propósitos, marcos teóricos y metodológicos, así como sus resultados. Por la densidad de los documentos y naturaleza 
de las preguntas que se hizo a cada fuente, se consideró pertinente tomar una muestra documental que permitiera a las investigadoras garantizar la cobertura textual completa de los contenidos.

El análisis de los contenidos de las fuentes se orientó por el propósito de aportar a la caracterización de la discusión sobre la formación de maestros para la educación inicial a partir de la producción investigativa de la UPN-Colombia. En aras de tratar dichos contenidos de manera sistemática y objetiva, la información de los documentos fue vaciada en una matriz (de contenido), por fuente, que posibilitó la identificación, separación y clasificación de la información, esto es, de los fragmentos de textos con temas o tópicos concernientes a la formación de maestros para la infancia. Posteriormente, la información de las matrices del con- junto de fuentes fue sometida a una lectura transversal que permitió identificar las tendencias en las discusiones sobre formación de maestros para la educación inicial.

Dichas tendencias se entienden como la evidencia de rasgos comunes en los planteamientos sobre formación de maestros para la educación inicial en las fuentes estudiadas, desde un análisis de contenido orientado por la lectura de los enunciados textuales, en torno a conceptos y categorías centrales del proyecto de investigación marco (investigación de la Red Educativa Universitaria de Conocimiento y Acción Regional-Reducar). Se trata de miradas provisorias derivadas del propósito y con las delimitaciones propias del criterio de selección de la fuente, es decir, es un primer acercamiento que exige investigaciones profundas.

\section{TeNDENCIAS EN LAS DISCUSIONES ACTUALES SOBRE LA FORMACIÓN DE MAESTROS}

El proceso inductivo seguido en la presente investigación posibilitó la emergencia de tres tendencias comprendidas como las principales disposiciones o preferencias en los temas y objetos de los veinte documentos indagados. Las tendencias presentan los hallazgos iniciales, parten de los datos $\mathrm{y}$, a partir de estos, las investigadoras proponen articulaciones con aspectos estructurales de la formación de maestros para la educación inicial en la UPN, como son las líneas de formación o de investigación. También, desde los datos, se proponen articulaciones con las políticas actuales o con los paradigmas o problemas en relación con la infancia y con la formación de maestros. Esta ruta implica que los referentes conceptuales de la indagación no se presentan en un apartado previo que antecede a los resultados, sino que el lector los hallará en diálogo con cada tendencia.

\section{AproximACIONES AL PENSAMIENTO, SABERES Y CONCEPCIONES DE LOS EDUCADORES INFANTILES EN FORMACIÓN Y EN EJERCICIO}

En esta tendencia se identifican dos entradas analíticas. La primera línea se aproxima al pensamiento y los saberes de los maestros en ejercicio a partir de sus expe- riencias, prácticas y reflexiones en relación con diferentes temas y problemáticas; la segunda se centra en las concepciones de los maestros en formación o en ejercicio acerca 
de problemáticas o campos de conocimiento que impactan la práctica pedagógica.

En esta primera línea se inscriben los trabajos de grado desarrollados por: Blanco, Murcia, Posso y Vargas (2014), Bautista, Méndez y Sequeda (2017), y Suárez, Getial e Ibagué (2014); también, tres informes de investigación de Pulido, Fandiño, Durán y Cruz (2016 y 2017), Fandiño, Pardo, González y Galeno (2019) y la tesis de maestría elaborada por Ramos (2017).

Los referentes que sustentan los trabajos de grado y las investigaciones abordan las creencias desde el campo de conocimiento denominado "pensamiento del profesor". La premisa fundamental es que las acciones de los maestros están relacionadas con sus creencias. Por tanto, se les concibe como profesionales en permanente reflexión sobre su quehacer; dicha reflexión se fundamenta tanto en la experiencia como en la teoría. Los principales antecedentes y autores que sustentan esta tendencia y que se citan en los trabajos de grado e informes de investigación mencionados en el párrafo anterior son: Shön, (1987), Clark y Peterson (1987), Marcelo (1987 y 1989), Contreras (1990), Pérez y Gimeno (1988), Fandiño (2007), Durán (2012), Reyes, Salcedo y Perafán (1999).

Es pertinente aclarar que hay dos enfoques en las investigaciones que se han desarrollado en el campo del pensamiento del profesor: un enfoque cognitivo, en la lógica de la solución de problemas y de la explicación de sucesos y acontecimientos (causa/ efecto) y, un enfoque alternativo que enfatiza en el sentido de las acciones de los maestros. En lo que respecta a los desarrollos y construcciones de los trabajos de grado, es evidente la búsqueda permanente por el sentido que otorgan los maestros a sus acciones, reconociendo la importancia de la interpretación de los sujetos y no la búsqueda de las causas. En consecuencia, el horizonte metodológico de los trabajos es cualitativo.

En relación con los saberes, estos son desarrollados desde los referentes de Tardif (2004) y Mercado (2002) (citados en dichos trabajos), para quienes la experiencia es la que pone en marcha el proceso de pensamiento: "El maestro "no piensa sólo con la cabeza", sino "con la vida", con lo que ha acumulado en términos de experiencia vital, en términos de bagaje de certezas" (Tardif, 2004, p. 75).

En este marco, los trabajos de grado hacen un acercamiento a elementos culturales, afectivos e históricos, como punto de partida para comprender la complejidad del pensamiento y de los saberes de los profesores. Así, emergen inquietudes sobre el juego, la comunicación y el lenguaje.

Los principales hallazgos de los trabajos de grado se relacionan con los marcos de comprensión de las intencionalidades y reflexiones que hacen las educadoras infantiles respecto a su quehacer y que las lleva a pensar en su lugar como maestras en formación, también se hace evidente la búsqueda por la identidad del educador infantil desde el pensamiento y los saberes de los maestros.

En lo que respecta a los informes de investigación, las preguntas se enfocan en el trabajo de campo orientado a la indagación de horizontes institucionales en colegios públicos (Pulido et al., 2016); y de profesoras, estudiantes de maestría y egresadas de la Licenciatura en Educación Infantil (Pulido et al., 2017). El propósito común es identificar, comprender y reflexionar acerca de las creencias del sentido de la educación ini- 
cial y su relación con la pregunta por el qué se enseña en educación inicial, para qué y cómo, con el fin de reconocer fortalezas y obstáculos en el enfoque de potenciamiento de desarrollo. Así mismo, se ahonda en el trabajo con maestras de educación inicial, con miras a caracterizar el saber sobre el desarrollo de los niños y niñas de primera infancia (Fandiño et al., 2019). Uno de los principales hallazgos de este estudio fue reconocer que los saberes de las maestras permiten ampliar el debate en torno a lo que significa valorar y medir el desarrollo de los niños con escalas elaboradas por psicólogos y médicos. Si bien es cierto que en esta investigación no se pretendió invalidar estas escalas, ni discutir si constituyen buenos instrumentos o no para el conocimiento de los niños, sí permitió reconocer que las maestras son interlocutoras válidas, o pares de otros profesionales para comprender el desarrollo de los niños.

En las construcciones teóricas, las investigaciones declaran la cercanía con el paradigma del pensamiento del profesor en cuanto a la relación creencias, saber y saberes (citan a Gary Fenstermacher, 1997). La relación se sustenta en el interés por comprender la práctica de los maestros bajo el supuesto que reconoce que el accionar de los seres humanos está más próximo al saber que al conocimiento, y que el saber, como forma amplia de intelección humana, está fuertemente arraigado a las dimensiones cognoscitiva, ética, estética y axiológica, según las finalidades que lo dinamicen. Esta sentencia está muy relacionada con lo que define a las creencias en términos de construcciones sociales a lo largo de la vida que permiten al maestro actuar en consecuencia con lo construido en su formación, con su experiencia y con su propia historia (Pulido et al., 2016).
En proximidad con los trabajos de grado, el saber de los maestros es una categoría transversal en las investigaciones, especialmente desde Tardif, en tanto se distancia de la perspectiva anglosajona, esto es, del sesgo individualista y mental, y enfatiza en sus particularidades sociales y subjetivas.

La tesis de maestría relacionada con esta línea analítica tuvo como objetivo comprender los procesos mentales de los profesores y la forma como estos influyen en su conducta; esto se fundamenta en los aportes de Fandiño (2004) y Jackson (1996), citados en Ramos (2017). Esta tesis asume la noción de creencias como "una proposición simple, consciente o inconsciente, inferida de lo que una persona dice o hace, capaz de ser percibida por la frase: -yo creo que". (Rockeach citado por Fandiño, 2004, en Ramos, 2017, p. 20). La tesis también retoma los aportes de Dewey (1994) en torno a las creencias, para quien estas abarcan "todas las cuestiones acerca de las cuales no disponemos de un conocimiento seguro, pero en las que confiamos lo suficiente como para actuar de acuerdo con ellas". (Ramos, 2017, p. 21)

La segunda línea analítica, diferente a la del pensamiento y saberes de los maestros, se centra en las concepciones de los maestros en formación o en ejercicio acerca de problemáticas o campos de conocimiento que impactan la práctica pedagógica, tales como las concepciones de infancia, el estatus de la infancia como sujeto de derechos y las concepciones de género.

El eje sobre el cual indagan tres trabajos de grado es el de concepciones, fundamentado por autores como Duarte (2011, 2013), citado por Contreras y Murcia (2017), quien las concibe como ideas creadas desde las vivencias y construcciones tanto individuales 
y colectivas, o desde autores como Andre Giordan (citado por las mismas autoras), para quien las concepciones son estructuras conceptuales o saberes compuestos desde las interrelaciones entre los contextos y las experiencias de los sujetos, susceptibles de mantenerse reconfigurarse, pues las dinámicas socio-históricas en las que el sujeto está inmerso conllevan reflexiones constantes. De manera similar, Laytón (2014) basa su trabajo de grado en la idea de concepción como el conjunto de ideas adoptadas o construidas por los sujetos, que pueden ser indagadas y que dan cuenta de comprensiones del mundo, así como de su actuar. Por su parte, Garzón, Nieto y Olmos (2019) centran la discusión en la perspectiva histórica de las concepciones ligadas a las nociones culturales y políticas de cada contexto y, por ende, con posibilidades de reconstruirse por parte de los individuos y grupos sociales.
Las concepciones se consideran influyentes en las acciones pedagógicas en la educación infantil. Las temáticas indagadas en esta segunda línea analítica y sus desarrollos investigativos visibilizan la intención de comprender, partir del paradigma cualitativo, las relaciones entre los discursos individuales y las prácticas pedagógicas, entre los referentes teóricos y legislativos y el que hacer e interacciones de los educadores con los niños y niñas.

El panorama que se evidencia en esta primera tendencia muestra dos posturas epistemológicas desde las cuales se realizan las investigaciones, la primera se sitúa en las creencias y saberes de los maestros y la segunda en sus concepciones. Vale la pena destacar que en ninguno de los trabajos, tesis e investigaciones se encontraron discusiones para diferenciar las perspectivas de creencias y concepciones.

\section{LAS PRÁCTICAS PEDAGógICAS Y LA FORMACIÓN DE PROFESIONALES REFLEXIVOS}

En esta tendencia, la práctica pedagógica se comprende como el lugar privilegiado en donde los maestros construyen y reconstruyen, problematizan y enriquecen saberes y cultura, así mismo, es objeto de estudio susceptible de problematizarse y enriquecerse. Para dar cuenta de esta tendencia se analizaron dos trabajos de grado, el de Andrade y Arias (2014) y el de Alfonso, Duque, González y Santana (2014); dos investigaciones orientadas a visibilizar las prácticas y promover escenarios de reflexión pedagógica que realizaron Rincón, Sierra y Galeano (2013) y Cano, Alberto, Revelo e Iquira (2018); y la tesis de maestría de Betancourt y López (2016), enfocada a la sistematización de una experiencia de formación.
El lugar epistemológico desde el cual se sustentan los trabajos de grado mencionados está estrechamente relacionado con los aportes de Shön, Giroux, y Martínez, Unda y Mejía, referenciados por Alfonso et al. (2014). El primer autor aporta sobre la formación de profesionales reflexivos, el segundo en relación con los maestros como intelectuales transformadores de las propias prácticas y procesos educativos y los últimos autores aportan desde su apuesta por el lugar de la experiencia y la reflexión en la construcción de saber pedagógico por parte de los maestros como trabajadores de la cultura.

Los referentes teóricos en relación con la práctica citados por Alfonso et al. (2014) 
y por Andrade y Arias (2014) son aquellos expresados por la UPN y por la Licenciatura en Educación Infantil, coincidentes con su carácter histórico y, por lo tanto, con un concepto dinámico, polisémico y resignificado según los contextos y realidades culturales, sociales, educativas, entre otras. De acuerdo con lo anterior, la práctica es un:

Proyecto pedagógico, cultural, social y político que propicia la construcción de saber pedagógico, a través de la generación de procesos de reflexión, resignificación e innovación de las experiencias pedagógicas, específicamente para la infancia colombiana, apoyadas en la investigación y contrastación de la diversidad de teorías, discursos y realidades de los diversos contextos educativos (Proyecto curricular de Educación Infantil-UPN, 2010, citado por las autoras).

La práctica ha sido indagada en el trabajo de grado de Andrade y Arias (2014) desde el marco metodológico de la Investigación Acción Pedagógica propuesto por Ávila. En él se hace alusión a que "es sin lugar a duda, una modalidad de investigación social, que busca explorar las intimidades de las prácticas pedagógicas, en el ámbito de la escuela" (Andrade y Arias, 2014, p. 57). Por otra parte, el trabajo de grado de Alfonso et al. (2014) se desarrolló desde el enfoque hermenéutico y el carácter interpretativo. Este les posibilitó reconocer en las prácticas diversas experiencias, comprenderlas y reconstruirlas, así como narrarlas para representar el mundo.

Los principales hallazgos estuvieron articulados a la experiencia investigativa y a los desarrollos pedagógicos. En consecuencia, los trabajos de grado destacan la evidente transformación de las propias prácticas pedagógicas a partir de los procesos de reflexión constante y colegiada, pues manifiestan que al tomar la práctica como objeto de estudio se desnaturaliza la cotidianidad y se moviliza el pensamiento respecto a las interacciones, sujetos, nociones, dudas, propósitos y la complejidad en cada uno de los escenarios educativos. Además, en estos trabajos se manifiesta que las reflexiones alcanzadas se acercan a los referentes del pensamiento crítico, puesto que las interrelaciones y cuestionamientos del quehacer en la práctica como maestras en formación, desde perspectivas teóricas y lecturas de las condiciones de los contextos, aportaron a su formación como maestras y, en algunas ocasiones, transformaron las realidades en las que participaron.

En la investigación de Rincón et al. (2013) se retoman los desarrollos y sustentos de los Espacios Enriquecidos de Comunicación y Lenguaje del ciclo de fundamentación de la Licenciatura de Educación Infantil, la noción de práctica pedagógica del programa, así como el sentido de la formación de maestros y la perspectiva interaccionista en la que se enmarcan los análisis sobre el conocimiento escolar y las reflexiones pedagógicas y didácticas. La perspectiva y los referentes desde los que se asume la investigación llevan a las investigadoras a afirmar que:

Las prácticas y saberes de los maestros no se limitan a un dominio instrumental de lo aprendido en la formación universitaria, sino que sus saberes son movilizados por los agentes educativos con los que interactúa en el espacio laboral, incluidos los niños. (...) Al enseñar, el docente se basa en varios tipos de juicios prácticos para orientar y estructurar su actividad profesional, tales como: valores morales o normas sociales; juicios provenientes de las tradiciones escolares y en su experiencia vivida. (Rincón et al., 2013) 
En cuanto a lo comunicativo, "no es suficiente con que niños y niñas estén en ambientes ricos lingüísticamente (programas de radio, televisivos, entorno con adultos que hablen entre sí) en los que se habla, sino que es esencial que hablen, que tengan interlocutores reales. El requisito para construir el lenguaje oral es hacerse un usuario efectivo del mismo y darle uso: debe manipularse, explorarse, jugar con él, valorarlo, notar que se necesita, que permite vivir y jugar, que tiene impacto en el medio en el que se está" (Reyes, 2010, citado por Rincón et al., 2013).

De otro lado, la investigación de Cano et al. (2018) apunta a diseñar una propuesta pedagógica desde la indagación guiada en relación con la actitud científica de niños de tres y cuatro años. Este ejercicio, si bien pasa por caracterizar la práctica del docente y su incidencia en el desarrollo de la actitud científica en los niños, se sustenta en las discusiones y construcciones teóricas en torno al trabajo de la ciencia con la infancia. Estas invitan al docente al desarrollo de la actitud científica, por ejemplo, desde los aportes de Wasserman (2004) y Garrido (2004), citados por Cano et al., 2018, como una oportunidad para enriquecer, fortalecer y complejizar el pensamiento del sujeto desde su edad inicial, a partir de acciones que "lo movilicen para para ampliar los referentes del mundo, así como a inquietarse frente a su realidad y a resolver situaciones o problemas que surgen en su cotidianidad" (Cano et al., 2018, p. 4).

En este trabajo se destaca el interés por reconocer al maestro, "como aquel que propicia situaciones de búsqueda y posibilita al niño preguntarse por el qué, cómo y por qué (...) donde se vea abocado a plantear hipótesis, comprobarlas e ir en búsqueda de respuestas a sus inquietudes". Estas ideas son cercanas a las reflexiones acerca de la alfabetización científica y las didácticas en educación infantil, en las que se reconoce el valor de las actividades experimentales ( $\mathrm{Ra}$ vanal, 2012, citado por Cano et al., 2018).

Otro modo de apreciar las relaciones entre la práctica y los referentes teóricos en las propuestas de formación se puede apreciar en la tesis de maestría de Betancourt y López (2016), quienes preguntan cómo se han transformado las prácticas profesionales de los educadores, hombres y mujeres de la SED que participaron en la línea de investigación de educación inicial y primera infancia del programa de Maestría en Desarrollo Educativo y Social UPN CINDE. Esta es una pregunta en torno a la formación docente ligada a discusiones sobre la infancia y orientada por referentes como Narodowsky (2011), sobre la relación maestro-niños, Hoyuelos (2009), sobre enseñanza e investigación y planteamientos de Freire (2004).

\section{Críticas al CurRículo y a Propuestas de Formación de LICENCIADOS EN EDUCACIÓN PARA LA INFANCIA}

En esta tendencia se encuentran cinco trabajos de grado que desarrollan Monroy, Velandia y Bustos (2014), Alberto y Murillo (2016), Romero (2016), Garzón et al. (2019) y Layton (2014); y una tesis de maestría realizada por Niño (2016).
Los referentes teóricos que sustentan los trabajos de grado se relacionan con los estudios de Vaillant (2007, citado por Romero, 2016) y Tedesco y Tenti (2002, citados por Monroy et al., 2014), quienes aportan referentes y tensiones sobre la identidad y 
prestigio de los maestros; también con los de Honoré (1980, citado por Garzón et al., 2019), en el sentido amplio de formación y la posibilidad de transformación de los individuos, los colectivos y, en consonancia, de la cultura. Otros referentes citados por Romero (2016) son los de Fandiño (2008) y Zabalza (2011) con sus trabajos sobre formación para la educación infantil. En relación con el currículo, Alberto y Murillo (2016) citan a Díaz Barriga (2009) y Torres (1994) por sus aportes sobre la concreción en el currículo de las intenciones y contenidos de la formación, así como la relevancia del currículo oculto.

Con base en los anteriores autores, los trabajos de grado realizan críticas frente a dos aspectos del currículo de la Licenciatura en Educación infantil que inició en el año 2.000. El primero se relaciona con la ausencia de contenidos y debates, por ejemplo, en torno a la interculturalidad, la sexualidad y el género. El trabajo de grado de Monroy et al. (2014) relaciona las ausencias en el currículo con lo expuesto por Juliao (2001), para quien, en las realidades siempre diversas, irrepetibles e inéditas, "el docente se enfrenta en la práctica a hechos no conocidos desde su formación" (Monroy et al., 2014, p. 106). Desde esta mirada, se realizan propuestas para que se incorporen ejes y contenidos en el currículo de los educadores infantiles.

El segundo aspecto tiene que ver con la preponderancia que se le otorga a la infancia, comprendida como una línea privilegiada en la formación de maestros y muy por encima de la que se hace sobre el maestro y los problemas inherentes a su formación, a su estatus y al malestar docente. Los trabajos de grado adicionan a la formación la necesidad no solo de saberes específicos desde las ciencias y disciplinas tradicionales, sino de campos de conocimiento transversales, por ejemplo, la sexualidad y el género, en los cuales se ven inmersos, interpelados y desprovistos de referentes para responder a las necesidades vividas desde las prácticas educativas en los diferentes escenarios.

Los trabajos de grado sobre el malestar docente (Romero, 2016) y la importancia de la interculturalidad (Alberto y Murillo, 2016) siguieron una ruta metodológica desde la perspectiva cualitativa. Por otra parte, hay una apuesta socio crítica de los trabajos de grado sobre sexualidad (Monroy et al., 2014) y género (Layton, 2014), desde la cual se pretende reconocer problemáticas y generar nuevos debates que incidan en la formación de educadores infantiles y aporten a la transformación social y cultural.

La presente tendencia puede interpretarse como una posibilidad de apropiación del proceso formativo de los educadores infantiles y de su agenciamiento para enriquecerlo. Sus reflexiones sobre las realidades que los convocan, desde los discursos teóricos y prácticos de la educación inicial, pretenden trascender la transformación de su currículo de formación, argumentando articulaciones y acercamientos con nuevas perspectivas en la formación; también, incorporando discursos emergentes que podrían entrar en diálogo con aquellos fundantes desde los cuales se ha configurado el currículo de la Licenciatura. Es relevante anotar que varias de las críticas al currículo de la Licenciatura en educación infantil condensadas en los trabajos de grado fueron consideradas en los debates y en la renovación de dicho programa en el año 2017.

La tesis de maestría referida a las críticas al currículo de formación (Niño, 2016), tuvo como objeto "los aspectos que constituyen la formación de los licenciados y li- 
cenciadas" (p. 30), a partir de la caracterización de las perspectivas teóricas, investigativas y de prácticas, así como de los compromisos sociales y políticos enunciados en cuatro programas de licenciatura ofertados en Bogotá. La revisión de documentos oficiales de las licenciaturas, producidos en el periodo 2009-2014, en particular los resultados de los procesos de autoevaluación, fue realizada desde un marco teórico amplio que deslinda la formación de maestros como una categoría social y una realidad profesional.

En la lógica de categoría social de Popkewitz (1987), citado en Niño (2016), se plantea que la formación de licenciados:

No funciona solo para producir maestros... sino que, como conjunto de procesos y de instituciones que es, refleja una cantidad de características culturales y estructurales tanto del sistema educativo, al que está vinculada de forma estrecha, como de la sociedad y de la economía a quienes debe su existencia. (p. 37)

Como realidad socioprofesional, se reconoce que la formación de maestros no es asunto meramente técnico, sino uno que se interrelaciona con "realidades más amplias, con mecanismos que afectan las relaciones sociales en las sociedades complejas, la división del trabajo, la estructura del control y el reparto del mismo en el sistema educativo" (Sacristán, 1987, citado en Niño, 2016, pp. 37-38). En esta vía, la formación, se reconoce ligada a modelos de escuela, a intereses y a valoraciones sociales acerca de la educación, en general, y de los niños, en particular.

Desde esta lógica, la tesis presenta las condiciones históricas y políticas que dieron lugar a la constitución del maestro y al ingreso de la mujer en el magisterio colom- biano y las discusiones y posturas en torno a la pedagogía, la práctica y la investigación en los procesos de formación. Esta fundamentación es complementada con una mirada panorámica a la infancia como campo de estudios y una contextualización acerca del "devenir de la educación de la infancia en Colombia" (Niño, 2016, p. 42).

Con este recaudo teórico, la autora identifica que lo constitutivo de la formación de licenciados para las infancias es difuso. En primer lugar, en el plano teórico, si bien se reconoce la pedagogía como referente esencial, no se evidencian desarrollos teóricos y conceptuales como resultado de investigaciones:

La producción de saber teórico y práctico en torno a la pedagogía y la pedagogía de la infancia, es un aspecto determinante en la formación profesional de licenciados en educación infantil; la ausencia de claridades y desarrollos pondría en situación de riesgo dicha formación profesional. (Niño, 2016, p. 266)

También, en lo que corresponde a la didáctica, la autora identifica la alusión a saberes específicos y formación disciplinar, como referentes para situar la especificidad del maestro en educación infantil:

Así, referencias al juego, la lúdica, la creatividad, música, arte plástico, cuerpo, enseñanza de la lengua escrita, educación matemática, se constituían desde las licenciaturas como posibles aspectos o saberes que han delimitado la educación de la infancia y, por tanto, la formación de los maestros en este campo. Saberes que ponen de manifiesto la tensión casi sempiterna de asumir la educación de la infancia como un espacio para la preparación para el ingreso a la básica primaria o como un espacio para el desarrollo del potencial expresivo de los niños y niñas. (Niño, 2016, p. 266) 
En todo caso, para la autora, "las referencias de estos saberes no permiten concluir que sean y se constituyan en asuntos que configuren la especificidad de un licenciado en educación para la(s) infancia(s)" ( $p$. 267), e identifica vacíos en torno a "cómo se recontextualizan los saberes que, provenientes de otras disciplinas, serán abordados en la educación de la infancia” (p. 267). Estas especificidades, de ser estudiadas, podrían ayudar a que "el maestro no se constituya en un 'todero profesional' que se aproxima a distintos saberes pero que en realidad no maneja ninguno a profundidad" (p. 267).

En segundo lugar, en el plano de la práctica, se evidencian dos enfoques como aspecto constitutivo en la formación de maestros para la infancia: como un campo de aplicación de las teorías y como "reconocimiento de las dimensiones sociales, políticas y culturales que constituyen la práctica en tanto realidad educativa" (Niño, 2016, p. 268). Estos enfoques encarnan las tensiones entre el sentido que se otorga a la teoría y a la práctica en la formación del maestro. A estas tensiones se suma "la proyección de la práctica en distintos contextos en los cuales se hace presente la infancia, (si bien), la formación de maestros parece haber trascendido o traspasado las fronteras del salón de clases" (Niño, 2016, p. 268). La autora deja abierta la pregunta acerca si los programas de formación y los escenarios de práctica preparan a sus maestros para los distintos contextos. Además, señala la necesidad de "abrir espacios de indagación que permitan penetrar las mismas prácticas para resolver, incluso, las diferencias entre práctica educativa y práctica pedagógica" (Niño, 2016, p. 269), en tanto se usan de forma indiferenciada en algunas de las licenciaturas indagadas.

En tercer lugar, se identifican diferentes abordajes: uno ligado a las orientaciones de las políticas que promocionan "la formación de una actitud investigativa, entendida ésta como una disposición o conducta favorable hacia la investigación"; otro positivista, "centrado en la aplicación del conocimiento científico a cuestiones educacionales" y un abordaje crítico orientado a "valorar y transformar su propio proceso autoeducativo" ( $p$. 269). En dichos abordajes no se expresan diferenciaciones entre investigación educativa y pedagógica y en todos los programas se hace alusión a la existencia de líneas de investigación, sin desarrollar "construcciones más particulares sobre los alcances, desarrollos, enfoques, métodos, teorías y aportes de estas líneas en la formación de sus licenciados" (p. 270).

Para Niño (2016), es claro que la investigación en la formación de licenciados en educación para la infancia se concibe y desarrolla en estrecha relación con la práctica y sus sentidos, desde un expreso compromiso social con la transformación de la realidad educativa de los niños en el país. Un compromiso loable, pero que debe extenderse a "la revaloración y empoderamiento de los licenciados como profesionales de la educación de la infancia, y en tanto tales, como sujetos de saber" (p. 276).

En cuarto lugar, se ahonda en la categoría de infancia, de niños y niñas, y de lo infantil en estos programas de formación. La mayoría de los abordajes se orientan por:

Una perspectiva del desarrollo integral en la que el rango etario es fundamental, en tanto permite la delimitación del campo de desempeño de los licenciados en educación para la infancia, y su respectiva organización y distribución en el sistema educativo en Colombia. (p. 270) 
Este punto de vista coexiste con "una aproximación a la infancia en su sentido plural, es decir, en la(s) infancia(s)" (p. 271), desde una lectura social y cultural, en la que se entiende lo infantil como "una construcción que hace referencia a un tiempo histórico y cultural construido por sujetos específicos con multiplicidad de experiencias" ( $p$. 271).

Aunque son perspectivas pertinentes y complementarias para la comprensión de lo que la infancia constituye como concepto y experiencia, para Niño (2016) se trata de referencias limitadas, en las que tienen peso los documentos de la política pública, con una tendencia a reconocer la infancia desde la carencia y la pobreza, por lo que los discursos sobre la enseñanza tienden a confundirse con lógicas de asistencia y atención. Así, se "genera el enrarecimiento de la figura de los maestros en educación de la infancia al corresponderles la tarea no solo de encargarse del desarrollo de procesos pedagógicos y educativos, sino también y con mayor énfasis, en la tarea de cuidar y atender las necesidades básicas de los niños" ( $p$. 271). Esta es una cuestión con la que se yuxtapone la responsabilidad de los adultos con los niños y las niñas a la especificidad de un proceso pedagógico institucional.

En lo que tiene que ver con la relación infancia y educación, para Niño (2016) es evidente que las diferentes nominaciones educación preescolar, educación inicial, educación para la primera infancia, educación infantil, abren la pregunta:

¿De qué tipo de educación se habla en relación con las infancias ante la configuración de un horizonte disperso que se propicia incluso desde las mismas políticas públicas que nominan de un modo y otro a esta educación; y ante la ausencia de desarrollos y argumentaciones teóricas por parte de las respectivas licenciaturas? (p. 72)

El cuestionamiento acerca de la educación para la infancia, de cara a la tensión cuidar - educar y a la perspectiva antiescolar, lleva a Niño (2016) a reconocer que:

Podría contribuir al reconocimiento de la pedagogía de la educación infantil como una línea de estudios en la que la especificidad de la educación de los niños es densa, exigente y abarcadora, por lo que no puede darse el lujo de romper con las relaciones con el sentido de preparación para la escuela, ni quedarse en las discusiones de lo innovador y lo tradicional. (p. 272)

Finalmente, un quinto elemento constitutivo en la formación de estos licenciados tiene que ver con las políticas públicas. Este es un aspecto transversal que evidencia cómo los marcos legales toman fuerza en las formas de nombrar la infancia, la educación y la formación de sus maestros de un modo particular. Así:

La diferencia entre una u otra licenciatura está en su grado de correspondencia y aceptación frente a este tipo de discursos. Lo clave de esta apreciación, consiste en afirmar que, con matices y acentos diferentes, ninguna de las licenciaturas se encuentra al margen de las políticas, pues a partir de ellas, justifican la existencia de sus programas y el sentido de la formación. (Niño, 2016, p. 273)

Justamente desde este referente legal y políticas de infancia se fortalece "la pareja atención y educación, que tiene implicaciones en la formación de maestros en educación para niños y niñas, pues al enfatizar en la atención y no en la educación, contribuye a la indiferenciación entre un docente y agente educativo" (Niño, 2016, p. 273). 
Ante la fuerza de este referente, Niño (2016) plantea:

Hoy se asiste a un desplazamiento en los modos de pensarse tanto la formación de maestros como de la educación de la infancia, pues en las circunstancias actuales, dicha formación y educación está en estrecha dependencia con los cambios y propuestas políticas que se agencian desde el Gobierno, sin mayores espacios para el debate, interlocución, análisis y reflexión entre universidades, Estado y sociedad. (p. 275)

Se puede inferir que, para Niño, recuperar esos debates pasa por estudiar "el devenir histórico en la formación de maestros en educación para la infancia en las últimas décadas del siglo veinte" (p.277), desde la misma experiencia de los programas, en tanto evidencias de las realizaciones, pero, también, de los vacíos. Se trata entonces de un estudio crítico, preocupado por "inventar un nuevo presente en la formación de maestros y en la educación de la infancia" ( $p$.
277). Una aproximación a lo anterior puede visibilizarse desde la pluralidad e interlocución presentes en la apuesta de renovación curricular del año 2017, de la Licenciatura en Educación Infantil de la Universidad Pedagógica Nacional. Allí se reconoció su historia desde varias perspectivas, así como sus logros; sin embargo, las críticas y retos fueron los puntos de análisis para pensar en condiciones de posibilidad, en lo nuevo, en lo diferente de la formación de maestros para la infancia.

Sin duda, el papel de la legislación y las políticas en el campo de formación de maestros merece atención especial. Si bien, en los trabajos de grado, investigaciones y tesis de grado retomadas en la presente investigación, estos aspectos no se analizan como problema central, todas los retoman para contextualizar, interrelacionar o dar sustento a sus problemáticas, así como para fundamentar las miradas a la infancia, la educación infantil y los maestros.

\section{Conclusiones}

Esta aproximación a la formación de los maestros desde preguntas e intereses investigativos ha posibilitado las siguientes reflexiones, problematizaciones y análisis.

- Los desarrollos investigativos tienen una estrecha relación con las líneas más antiguas de la Licenciatura en educación infantil de la UPN, a saber, juego, cultura y crecimiento, que surge en 1987, y pensamiento y creencias del profesor en educación infantil, creada en 1999. Estas líneas hacen parte del Grupo de Investigación Educación Infantil Pedagogía y Contextos (Fandiño, Duran y Pulido, 2018).
También tienen estrecha relación con los espacios enriquecidos de lúdica y psicomotricidad, comunicación y lenguaje, exploración de medio y educación matemática, desde los cuales se articulan aspectos disciplinarios, pedagógicos, didácticos e investigativos. Ello ha movilizado, por una parte, procesos de formación, como se puede ver en los trabajos de grado y las tesis de maestría y, por otra parte, los proyectos de investigación y las propuestas de acompañamiento y cualificación a maestros en ejercicio. Estos procesos han sido fundamentales para la proyección social de la Universidad Pedagógica 
Nacional. Sin embargo, se vislumbra la importancia de ahondar en las preguntas acerca del qué enseñar y cómo enseñar en el contexto escolar, promoviendo la equidad de las oportunidades de aprendizaje y experiencias de enseñanza en el nivel de la educación inicial.

- La formación de maestros no está desprovista del estudio riguroso del pensamiento y los saberes del profesor desde los acumulados que brinda la experiencia y la propia práctica (Zabalza, 1988; Porlain, Rivero y Martín del Pozo, 1997; Spodek y Rucinski, 1985). Hoy viene tomando fuerza este tipo de investigaciones que se enfocan en la práctica del maestro en formación y en ejercicio, como posibilidades para reconocer que los saberes y las creencias son construcciones sociales producto de un devenir histórico, que se relacionan de forma directa con las concepciones de infancia, educación infantil, maestro o escuela y que aportan a la reconfiguración de prácticas que interpelan el lugar de la enseñanza y la pedagogía en educación infantil.

- De las fuentes analizadas, se puede colegir que la formación de educadores infantiles en la UPN está atravesada por la posibilidad de construir conocimiento profesional a partir de inquietudes por el sentido de lo educativo, lo pedagógico, lo didáctico en clave de trayectorias educativas, así como por la intención de comprender categorías que fundamentan las prácticas educativas en la actualidad. Se visualiza la necesidad de comprender o desnaturalizar discursos teóricos y prácticos de los maestros desde sus propias reflexiones y valoraciones. De este modo, los temas y objetos de investigación en el terreno de la educación infantil evidencian el compromiso con la producción de conocimiento, con la certeza de que el maestro es productor de saber en articulación con la historia vital, la identidad o las experiencias (Contreras, 2010).

- La formación de maestros en cuanto a la producción investigativa está muy relacionada con la práctica, lo que puede reconocerse como una fortaleza de su formación en la educación inicial. No obstante, ese realce de lo práctico debe ser objeto de reflexión sobre las relaciones con el plano teórico. Estas relaciones no pueden perderse de vista, no puede ganar terreno la perspectiva desde la cual se minimiza la relevancia de la teoría en el trabajo pedagógico con niños y niñas pequeños. De ahí que se vislumbre la importancia de interrogar continuamente la fundamentación teórica de la práctica, en tanto condición que orienta el estudio, análisis y discusión en torno a los campos relacionados con la infancia y la educación infantil, así como las miradas críticas y reflexivas sobre el quehacer del maestro y el currículo.

- La investigación identificó varias categorías presentes en la práctica pedagógica de la Universidad Pedagógica Nacional, entre estas, la experiencia y la reflexión. Se requiere continuar el estudio de estas categorías con mayor desarrollo teórico conceptual, en tanto están relacionadas con campos diferentes, como el de la educación, la pedagogía, la didáctica y los saberes específicos. Ello implica acercamientos contextualizados y críticos a referentes en el campo de la pedagogía, la filosofía, la sociología y la psicología, por ejemplo. 
- Finalmente, el análisis permite considerar la formación de maestros para la educación infantil como una realidad socio profesional en la que se juegan valoraciones sociales acerca del maestro, la escuela, la infancia y los niños, así como disputas por el reconocimiento del estatus profesional de los educadores infantiles. En esta vía, se reconoce la necesidad de ahondar en el campo de estudios en torno a las condiciones históricas, políticas y académicas que instituyen y problematizan tanto el lugar del maestro de educación infantil en sociedades como la colombiana, como su formación inicial y permanente. Estas cuestiones están ligadas a preguntas por la educación infantil como campo conceptual y profesional, por la infancia como concepto y como experiencia, por las realidades educativas de los niños y niñas en matrices socioculturales específicas, por lo que implica educar a los niños pequeños y por lo que debe saber ese sujeto profesional que pretende educar a las infancias. Estos aspectos son esenciales para que la Universidad Pedagógica Nacional responda con la contundencia necesaria a la formación de maestros para la educación inicial, en un país que intenta dar el paso a la reconciliación de la sociedad y, por ende, a la construcción de un presente y un futuro con condiciones dignas de vida para todos y, en especial, para las nuevas generaciones.

\section{REFERENCIAS}

Alfonso, Y., Duque, L., González, E., y Santana, J. (2014). Aproximaciones de cuatro maestras en formación al saber pedagógico, en el marco de la expedición por las prácticas (tesis de pregrado). Universidad Pedagógica Nacional, Bogotá, Colombia. http://hdl.handle. net/20.500.12209/2434

Alberto, D., y Murillo, A. (2016). Interculturalidad y educación intercultural, incidencia en la formación de maestros. Sugerencias para la renovación curricular del programa de la Licenciatura en educación infantil de la UPN (tesis de pregrado). Universidad Pedagógica Nacional, Bogotá, Colombia. http://hdl.handle.net/20.500.12209/2500

Andrade, M., y Arias, A. (2014). Los textos orales en la primera infancia: una experiencia investigativa desarrollada con maestras egresadas en la Escuela Maternal de la UPN (tesis de pregrado). Universidad Pedagógica Nacional, Bogotá, Colombia. http://hdl.handle.net/20.500.12209/2447

Bautista, J., Méndez, K., y Sequeda, A. (2017). Incidencia de las creencias de maestros y maestras de grado transición y básica primaria, en la enseñanza de la lengua extranjera, inglés (tesis de pregrado). Universidad Pedagógica Nacional, Bogotá, Colombia. http://hdl.handle. net/20.500.12209/9948

Blanco, J., Murcia, L., Posso, A., y Vargas, D. (2014). Maestras, juego, vivencias: una aproximación a las creencias sobre el juego de seis maestras del Colegio Aquileo Parra I.E.D. (tesis de pregrado). Universidad Pedagógica Nacional, Bogotá, Colombia. http://hdl.handle.net/20.500.12209/2437 
Betancourt, L., y López, A. (2016). Sistematización del proceso formativo de maestros y maestras de la Secretaría de Educación que participaron en la Linea de Investigación de Educación Inicial y Primera Infancia de la Maestría en Desarrollo Educativo y Social UPN - CINDE (tesis de maestría). Universidad Pedagógica Nacional, Fundación Centro Internacional de Educación y Desarrollo Humano, CINDE, Bogotá, Colombia. http://hdl.handle.net/20.500.12209/723

Cano, E., Alberto, D., Revelo, L., e Iquira, J. (2018). Desarrollo del pensamiento científico en niños de tres y cuatro años de la Escuela Maternal (informe final de investigación). Centro de Investigaciones de la Universidad Pedagógica Nacional, Bogotá, Colombia.

Contreras, J. (2010). Investigar la experiencia educativa. Madrid: Morata.

Contreras, Y., y Murcia, Y. (2017). Acercamiento a las concepciones de infancia de algunos maestros en formación de la Licenciatura en educación infantil de la Universidad Pedagógica Nacional (tesis de pregrado). Universidad Pedagógica Nacional, Bogotá, Colombia. http://hdl.handle. net/20.500.12209/2415

Fandiño, Durán, y Pulido (2018). Trayectorias y rutas posibles para la investigación en educación infantil. Bogotá, Colombia: Fondo Editorial de la Universidad Pedagógica Nacional. http://editorial.pedagogica.edu.co/docs/files/CIUPLibro\%206\%20final.pdf

Fandiño, G., Pardo, A., González, K., y Galeano, J. (2019). Caracterización del saber de las maestras de educación inicial sobre los niños de primera infancia a su cargo (informe final de investigación). Centro de Investigaciones de la Universidad Pedagógica Nacional, Bogotá, Colombia.

Garzón, A., Nieto, G., y Olmos, D. (2019). La infancia como sujeto de derecho: una mirada desde los procesos de formación en los maestros/as de educación infantil de la Universidad Pedagógica Nacional (tesis de pregrado). Universidad Pedagógica Nacional, Bogotá, Colombia. http://hdl. handle.net/20.500.12209/10238

Layton, J. (2014). Mujeres tejiendo historia. Aproximación a las concepciones de género de las maestras en formación de primer semestre de la Licenciatura en Educación Infantil de la UPN (tesis de pregrado). Universidad Pedagógica Nacional, Bogotá, Colombia.

Monroy, D., Velandia, I., y Bustos, J. (2014). La formación de los docentes en relación con la educación para la sexualidad de la primera infancia dentro de la Licenciatura de educación infantil de la Universidad Pedagógica nacional (tesis de pregrado). Universidad Pedagógica Nacional, Bogotá, Colombia. http://hdl.handle.net/20.500.12209/2436

Niño, S. (2016). Lo constitutivo de la formación de profesores de educación infantil: Apuntes para comprender la educación de la(s) infancia(s) (tesis de maestría. Universidad Pedagógica Nacional, Bogotá, Colombia. http://hdl.handle.net/20.500.12209/1059

OEI. (2000). X Conferencia Iberoamericana de Educación (Ciudad de Panamá, Panamá, 3 y 4 de julio). En línea: http://www.oei.es/historico/xcie.htm

Porlán, R., Rivero, A., y Martín del Pozo, R. (1997). Conocimiento profesional y epistemología de los profesores I: Teoría, métodos e instrumentos. Enseñanza de las ciencias. Revista de investigación y experiencias didácticas, 15 (2), 155-71. https://www.raco.cat/index.php/Ensenanza/article/ view/ 
Pulido, J., Fandiño, G., Durán, S., y Cruz, E. (2017). Creencias sobre la educación inicial en estudiantes de la maestría en Estudios en Infancias y egresadas de la Licenciatura en Educación Infantil de la Universidad Pedagógica Nacional (informe final de investigación). Centro de Investigaciones de la Universidad Pedagógica Nacional, Bogotá, Colombia.

Pulido, J., Fandiño, G., Durán, S., y Cruz, E. (2016). Creencias sobre la educación inicial en tres instituciones educativas distritales (informe final de investigación). Centro de Investigaciones de la Universidad Pedagógica Nacional, Bogotá, Colombia.

Universidad Pedagógica Nacional de Colombia (2010). Programa curricular de Educación Infantil. Informe de Evaluación con fines de renovación de la acreditación de calidad. Documento interno.

OREALC/UNESCO (2016). Estado del arte y criterios orientadores para la elaboración de políticas de formación y desarrollo profesional de docentes de primera infancia en América Latina y el Caribe. Chile. Disponible en https://unesdoc.unesco.org/ark:/48223/pf0000245157

Ramos, B. (2017). Lo que está en juego, en el juego: Creencias sobre el juego de una maestra del ciclo de educación inicial y una maestra del ciclo de básica de la IED Montebello y el lugar de ellas en el mismo (tesis de maestría). Universidad Pedagógica Nacional, Bogotá, Colombia. http://hdl. handle.net/20.500.12209/7821

Rincón, C., Sierra, M., y Galeano, J. (2013). Prácticas pedagógicas de las egresadas del proyecto curricular de educación infantil de la UPN que promueven la oralidad en niños menores de cinco años (informe final de investigación). Centro de Investigaciones de la Universidad Pedagógica Nacional, Bogotá, Colombia.

Romero, Y. (2016). La importancia de las capacidades socioafectivas para prevenir el malestar docente en la formación de los y las estudiantes de la Licenciatura en educación infantil de la Universidad Pedagógica Nacional (tesis de pregrado). Universidad Pedagógica Nacional, Bogotá, Colombia. http://hdl.handle.net/20.500.12209/2406

Spodek, B., y Rucinski, E. A. (1985). A study of early chidhood teachers beliefs: preschool teachers. Paper presented at anual meeting of AERA. Chicago.

Suárez, J., Getial, T., e Ibagué, Y. (2014). Acompañamiento en educación infantil: una experiencia en torno a la oralidad y saberes del maestro (tesis de pregrado). Universidad Pedagógica Nacional, Bogotá, Colombia. http://hdl.handle.net/20.500.12209/7723

Tardif, M. (2004). Los saberes del docente y su desarrollo profesional. Madrid: Narcea, S.A.

Zabalza, M. A. (1988). Condiciones metodológicas en el estudio del pensamiento del profesor. Los autoinformes. En: C. Marcelo (ed). Avances del pensamiento de los profesores. Sevilla: Serv. Public. Universidad de Sevilla. 